\title{
Relationship between TLR4 and CCL2 expression and recurrent spontaneous abortion
}

\author{
P. Li ${ }^{1,2}$, H.L. Wu' ${ }^{2}$ and B.H. Dong ${ }^{1}$ \\ ${ }^{1}$ Department of Gynecology and Obstetrics, Qilu Hospital, \\ Shandong University, Jinan, China \\ 2Department of Gynecology and Obstetrics, \\ Affiliated Hospital of Taishan Medical University, Taian, China \\ Corresponding author: B.H. Dong \\ E-mail: marktwinoun@163.com \\ Genet. Mol. Res. 15 (1): gmr.15016882 \\ Received August 28, 2015 \\ Accepted October 24, 2015 \\ Published January 8, 2016 \\ DOI http://dx.doi.org/10.4238/gmr.15016882
}

ABSTRACT. The increasing incidence of recurrent spontaneous abortion (RSA) severely affects women's health. The involvement of the immune system during pregnancy and its contribution to RSA draw researchers' attention. Both Toll-like receptor 4 (TLR4) and chemokine (C-C motif) ligand 2 (CCL2) have been linked to various pregnancy disorders. In this study, we quantified TLR4 and CCL2 levels in a case-control study in order to elucidate the correlation between these factors and RSA, and the potential to use them as disease markers. A total of 36 RSA patients and 36 healthy control individuals were recruited for the donation of decidual and chorionic tissues and venous blood samples. Fluorescent quantitative-polymerase chain reaction was used to measure mRNA levels and enzyme linked immunosorbent assay was used to quantify serum levels of TLR4 and CCL2. RSA patients had higher TLR4 and CCL2 mRNA levels compared to controls $(P<0.05)$. Serum levels of the two factors were also significantly higher in RSA patients than in the control group $(P<0.05)$. Positive correlations were found between 
serum levels and tissue mRNA levels of TLR4 and CCL2. In conclusion, both TLR4 and CCL2 were closely related to the occurrence of RSA, suggesting that serum TLR4 and CCL2 levels could be used as indices for monitoring RSA in pregnant women.

Key words: Recurrent spontaneous abortion; Toll-like receptor 4; Chemokine (C-C motif) ligand 2

\section{INTRODUCTION}

Recurrent spontaneous abortion (RSA), also known as habitual abortion, refers to the occurrence of multiple spontaneous abortions at less than 20 weeks of pregnancy (Ford and Schust, 2009). As a common cause of female infertility, RSA has complex etiological factors, including chromosome abnormality, immune dysfunction, acquired infection, endocrine disease, anatomical defects, and environmental toxicity. However, more than half of the RSA cases have no definitive cause and are classified as unexplained RSA.

With the advancement of medical research, it is known that pregnancy is dependent on innate immunity, making pregnant immunology a newly emerging and exciting research topic. Thus far, most scholars have attributed spontaneous abortion to the immune imbalance between mother and fetus, resulting in the rejection of fetal tissues and miscarriage (Takeshita, 2004; Koga and Mor, 2010). Toll-like receptors (TLRs) are a family of newly discovered receptors that are crucial for innate immunity and are highly conserved in all invertebrates and vertebrates (Pevsner-Fischer et al., 2007). It has been reported that TLRs are involved in the maintenance of pregnancy and occurrence of pregnancy disorders (Amirchaghmaghi et al., 2013; Koga et al., 2014). Both amnion cells and trophocytes at the maternal-fetal interface may be involved in innate immunity by the activation of TLRs, which then affects the outcome of pregnancy. TLR4 may lead to the release of inflammatory factors via activating nuclear transcription factor $\mathrm{KB}$. Several studies have reported elevated TLR4 expression in inflammatory cells in inflammation-triggered premature delivery (Beijar et al., 2006). Therefore, a potential relationship may exist between TLR4 and occurrence of RSA.

Chemokine (C-C motif) ligand 2 (CCL2), also called monocyte chemotactic protein-1 (MCP1), mainly exerts its functions in recruiting and activating mononuclear cells. CCL2 is produced by various cell types, including uterus endothelial cells, which secrete high amounts of chemokines in the first trimester of pregnancy at the maternal-fetal interface (Drake et al., 2002). It has been demonstrated that chemotactic factors participate in the immune response at the maternal-fetal interface during the implantation of the embryo (Hannan et al., 2004). In addition, further studies revealed significantly elevated expression of CCL2 in decidual and chorionic tissues in women who delivery prematurely when compared to normal pregnant controls (Zhang et al., 2012). As a result, CCL2 expression may also mediate the occurrence of RSA.

We performed a case-control study examining 36 RSA patients and 36 normal pregnant women. Decidual and chorionic tissues were quantified for TLR4 and CCL2 mRNA using fluorescent quantitative polymerase chain reaction (qPCR) and further correlation analysis was performed, thus providing a new method for prognostic RSA prediction. In addition, serum TLR4 and CCL2 levels were measured in venous blood to analyze the correlations with their respective mRNA levels. These indices may provide a potential clinical application in the early diagnosis of RSA. 


\section{MATERIAL AND METHODS}

\section{Patient information}

This study was pre-approved by the Ethics Committee of the Affiliated Hospital of Taishan Medical University (China) and written consent was obtained from all patients. A total of 36 RSA patients who were admitted to the hospital for dilatation and curettage after spontaneous abortion were included in this study. The inclusion criteria were modified from previously reported standards (Lin and Qiu, 2010). The patients were included if they had 1) no more than two spontaneous abortions; 2) no systematic disease; 3) a normal menstrual period; 4) a normal karyotype (their partner also had a normal karyotype); 5) partners with normal semen; 6) negative results for autoanti-sperm, anti-nucleus, or anti-phospholipid antibodies; 7) no anatomical defects or deformation of reproductive organs; and 8) no infection of the reproductive tract. Another 36 individuals who were currently pregnant or had undergone induced abortion due to medical reasons other than those mentioned above were recruited in the control group. The inclusion criteria of the control group were that the individuals must 1) not be in their first pregnancy; 2) have no history of spontaneous abortion or stillbirth; 3) have no systematic disease; 4) B-mode ultrasound confirmed intrauterine pregnancy; 5) have no infection of the reproductive tract; 6) have no bleeding or abdominal pains since menopause; and 7) have no genetic, endocrinological, or anatomical abnormalities. Decidual and chorionic tissues were obtained during surgery and $3 \mathrm{~mL}$ venous blood was collected before surgery. No significant difference existed between disease (RSA) and control groups regarding age, the number of pregnancies, and the days in menopause (Table $1 ; \mathrm{P}>0.05$ ).

Table 1. General information about patients.
\begin{tabular}{lcccc}
\hline Group & N & Age (years) & No. of pregnancies & Menopause days \\
\hline Disease & 36 & $29.31 \pm 3.43$ & $2.82 \pm 0.56$ & $50.62 \pm 4.23$ \\
Control & 36 & $28.64 \pm 3.96$ & $3.02 \pm 0.62$ & $49.18 \pm 5.27$ \\
$t$ value & & 0.77 & 1.44 & 1.28 \\
P value & & 0.22 & 0.08 & 0.10 \\
\hline
\end{tabular}

\section{RNA extraction and cDNA synthesis}

Tissue samples were grinded by mortar and pestle over liquid nitrogen and rinsed in $1 \mathrm{~mL}$ Trizol (Invitrogen, USA). After $10 \mathrm{~min}, 0.2 \mathrm{~mL}$ chloroform was added followed by mixing. The mixture was then centrifuged for $15 \mathrm{~min}$ after incubation for $5 \mathrm{~min}$. Supernatants were transferred to new tubes containing equal volumes of isopropanol. After mixing, another 10-min centrifugation was performed, following which the supernatants were discarded. RNA pellets were then washed using $1 \mathrm{~mL} 75 \%$ ethanol, followed by 5-min centrifugation. RNA was dried at room temperature and resuspended in Tris-EDTA buffer. Purity and concentration of RNA were determined by spectrophotometer analysis. Then, $1 \%$ agarose gel electrophoresis was used to determine the intact conformation of RNA (Figure 1). In vitro reverse transcription was performed using $1 \mathrm{mg}$ RNA as the template (TaKaRa, Japan) following the manufacturer instructions. A total volume of $10 \mu \mathrm{L}$ was used for reverse transcription performed using a PCR cycler (ABI, USA) with the following protocol: $37^{\circ} \mathrm{C}$ for $15 \mathrm{~min}$ followed by $85^{\circ} \mathrm{C}$ for $5 \mathrm{~s}$. CDNA was kept at $-20^{\circ} \mathrm{C}$ for further use. 


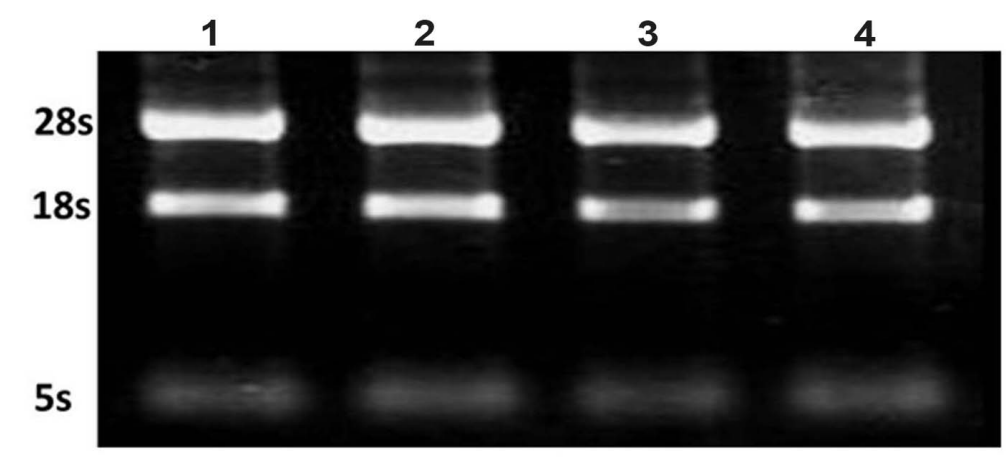

Figure 1. Gel electrophoresis of total RNA extracted: 28,18 , and 5 S ribosomes are labeled. Lanes $1-4=$ Partial gel electrophoresis samples.

\section{Real-time qPCR}

The reaction mixture was prepared as follows: $5 \mu \mathrm{L}$ SYBR Green (Toyobo, Japan), 0.5 $\mu \mathrm{L}$ cDNA template, $0.5 \mu \mathrm{L}$ primer $(10 \mu \mathrm{M})$ and $4 \mu \mathrm{L} \mathrm{ddH}_{2} \mathrm{O}$. The reaction was performed on a fluorescent qPCR cycler (ABI) using the protocol: denaturation at $95^{\circ} \mathrm{C}$ for $15 \mathrm{~s}$ and annealing and elongation at $60^{\circ} \mathrm{C}$ for $60 \mathrm{~s}$ (repeated for $40 \mathrm{cycles}$ ). Each experiment was performed in triplicate using $\beta$-actin as the internal reference. Primer sequences are listed in Table 2.

\begin{tabular}{ll}
\multicolumn{1}{c}{ Table 2. Primers used in qPCR. } & \\
\hline Target gene & Primer sequence $\left(5^{\prime} \rightarrow 3^{\prime}\right)$ \\
\hline TLR4 & Forward: TGGATACGTTTCCTTATAAG \\
& Reverse: GAAATGAGGCACCCCTTC \\
CCL2 & Forward: CAATCAATGCCCCAGTCACC \\
$\beta$-actin & Reverse: CCTGAACCCACTTCTGCTTG \\
& Forward: GCACTCTTCCAGCCTTCC \\
\hline
\end{tabular}

\section{Enzyme-linked immunosorbent assay (ELISA)}

Serum TLR4 and CCL2 levels were measured using an ELISA kit (R\&D, USA) following the manufacturer instructions. In brief, standard samples with serial dilutions and serum were added onto a 96-well plate in triplicate. After incubation for $120 \mathrm{~min}$ at $37^{\circ} \mathrm{C}$, the supernatant was removed from the well and washed with washing buffer for $3 \mathrm{~min}$. After washing 3 times, working solution $A$ was added into each well for $60 \mathrm{~min}$ at $37^{\circ} \mathrm{C}$. Working solution $\mathrm{B}$ was then added followed by another round of incubation. The reaction was stopped by adding quenching buffer (50 $\mu \mathrm{L}$ ). Optical density values at $450 \mathrm{~nm}$ were measured using a micro-plate reader (Bio-Tek, USA). Serum levels were deduced from the standard curve, which was plotted based on serially diluted standard samples.

\section{Statistical analysis}

The SPSS 13.0 software package (SPSS Inc., USA) was employed to perform all statistical 
analysis. The comparison of means was performed by an independent two-sample Student $t$-test. Correlation analysis was completed using the Pearson method. All data are reported as means \pm standard deviation (SD). Statistical significance was defined as $P<0.05$.

\section{RESULTS}

\section{TLR4 and CCL2 mRNA levels in tissues}

The relative levels of TLR4 and CCL2 mRNA in decidual and chorionic tissues were analyzed by the $2^{-\Delta \Delta C t}$ method. As shown in Figure 2, TLR4 mRNA levels were significantly higher in both chorionic or decidual tissues in the RSA disease group compared to the control group ( $P<$ 0.05). The levels of CCL2 mRNA were also significantly higher in both tissue types of RSA patients compared to the control group $(P<0.05)$.
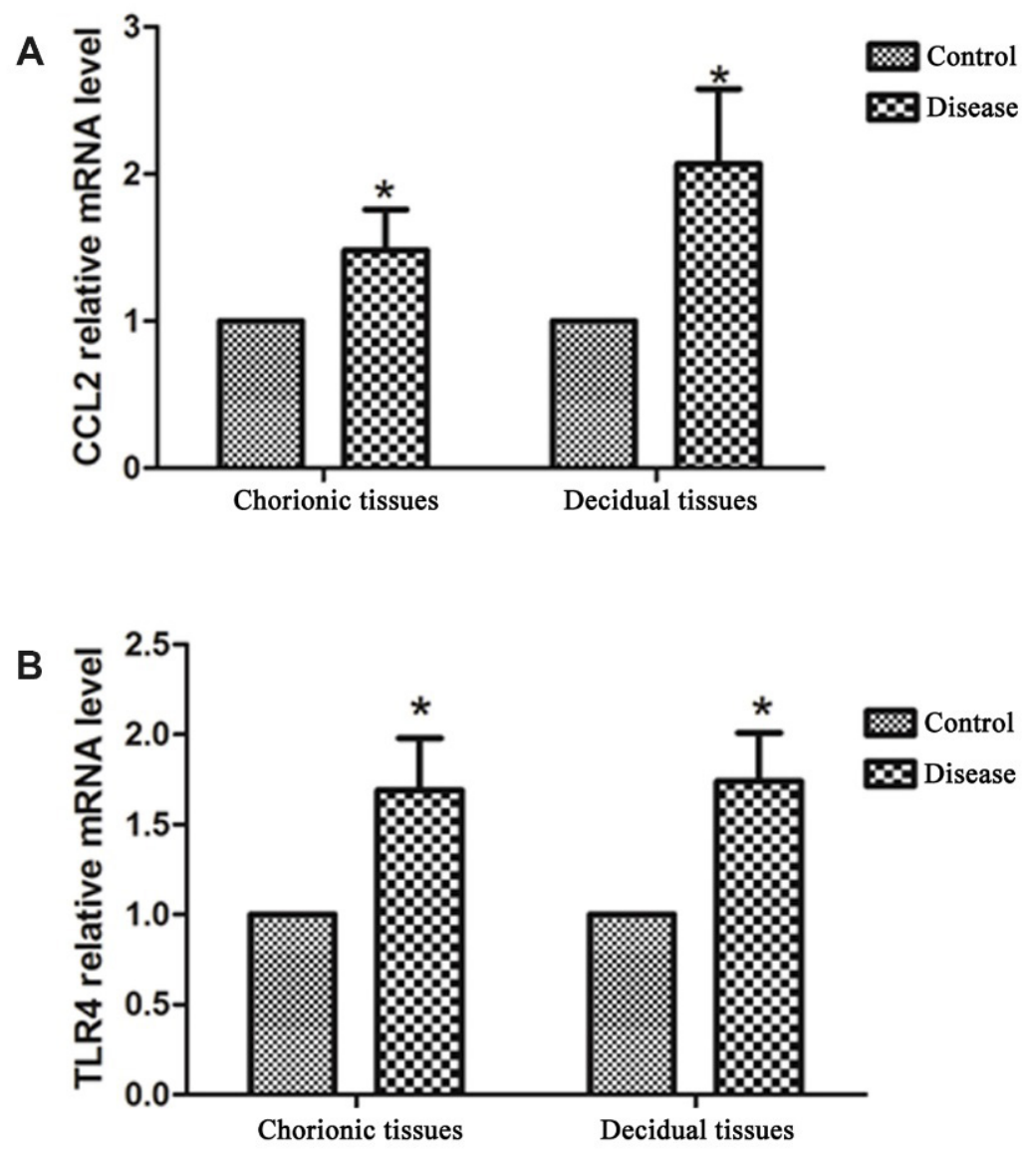

Figure 2. mRNA levels of CCL2 (A) and TLR4 (B) in decidual and chorionic tissues. The mRNA levels of both genes were significantly elevated in both tissue types of RSA (disease) patients compared to the control group. ${ }^{*}<0.05$ compared to the control group. 


\section{Serum TLR4 and CCL2 levels}

The serum levels of TLR4 and CCL2 were analyzed by ELISA. As shown in Table 3 and Figure 3, both factors had significantly elevated serum levels in the RSA disease group compared to the control group $(P<0.05)$.

Table 3. Serum levels of TLR4 and CCL2.
\begin{tabular}{lcc}
\hline Group & TLR4 level $(\mathrm{ng} / \mathrm{mL})$ & CCL2 level (ng/mL) \\
\hline Disease & $1.68 \pm 0.38$ & $79.93 \pm 6.63$ \\
Control & $0.80 \pm 0.27$ & $52.54 \pm 9.98$ \\
- -value & 26.83 & 13.72 \\
P value & $<0.001$ & $<0.001$ \\
\hline
\end{tabular}

A

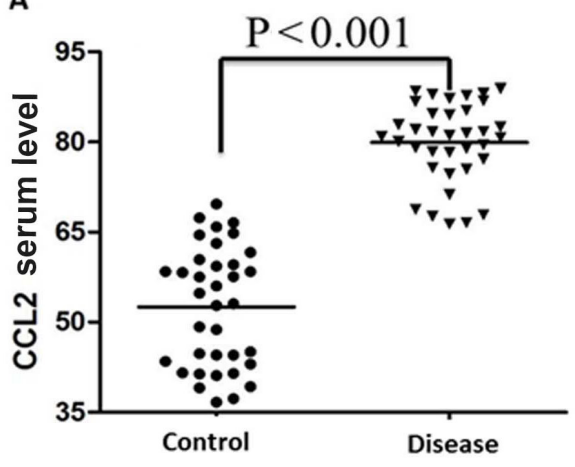

B

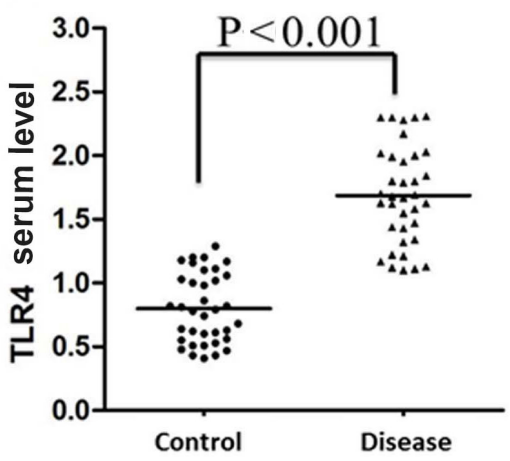

Figure 3. Scatter plot of serum CCL2 (A) and TLR4 (B) levels. Both CCL2 and TLR4 serum levels were significantly elevated in the RSA (disease) group compared to the control group.

\section{Correlation analysis between mRNA and serum levels of TLR4 and CCL2}

We next employed the Pearson correlation analysis to test if there was a significant relationship between serum concentrations and decidual/chorionic tissue mRNA levels of TLR4 or CCL2. Our results showed a positive correlation between serum and decidual or chorionic tissue mRNA TLR4 levels ( $r=0.541$ for decidual and 0.623 for chorionic tissue; $P<0.05$ in both cases). CCL2 showed similar patterns as serum levels were positively related to decidual/chorionic mRNA levels ( $r=0.745$ for decidual and 0.698 for chorionic tissue; $P<0.05$ in both cases).

\section{DISCUSSION}

Pregnancy is a complex immune process involving both the protection of the mother's body against external pathogens and the tolerance of the fetus, which carries antigens from the biological father (Gobert and Lafaille, 2012). This balance between immune reaction and tolerance is fundamental for the survival of the fetus. Therefore, it is common to attribute miscarriage to the dysfunction of the maternal immune system by rejection of the fetus. Therefore, attention should be focused on immune balance at the maternal-fetal interface, in which the maternal side 
consists of decidual immune and matrix cells while the fetal side includes chorionic trophoblasts. This study used decidual and chorionic tissues for quantification of TLR4 and CCL2 mRNA levels, both of which are known to be involved in the immune response during pregnancy. The serum levels in blood of these two factors were also examined to identify their potential as biological markers.

As an important member of the TLR family, TLR4 can directly activate innate immunity to release inflammatory mediators such as interferon- $\alpha$ (INF- $\alpha$ ), INF- $\gamma$, interleukin-1 (IL-1), IL-6 and IL-8 (Kopp and Medzhitov, 1999), all of which work as the primary defense line in innate immunity (Dunzendorfer et al., 2004). The expression of TLR4 at the maternal-fetal interface exerts a crucial role in maintaining immune tolerance and protecting against external pathogens. This study found elevated TLR4 expression in the chorionic and decidual tissues of RSA patients compared to normal pregnant women, suggesting the involvement of TLR4 in the occurrence of RSA. To our knowledge, there have been no studies reporting the correlation between TLR4 and RSA occurrence. A study in women who have had miscarriages found elevated expression of tumor necrosis factor- $\alpha$ (TNF- $\alpha$ ), INF- $\gamma$ and INF- $\beta$ and decreased expression of IL-10, while no T helper type 1 (Th1) cytokines were identified in the control group. Interestingly, the control group also exhibited elevated IL-10 levels (Hill and Choi, 2000). Further studies also revealed significantly lower amounts of Th1 cytokines such as TNF- $\alpha$ and INF- $\gamma$ in women predisposed to spontaneous abortion (Chaouat et al., 1995). The expression of TLR4 may facilitate secretion of inflammatory cytokines such as TNF- $\alpha$ and INF- $\gamma$; elevated expression of these cytokines in the placenta plays a crucial role in inducing trophocyte apoptosis (Saini et al., 2011). Therefore, TLR4 may play a role in RSA pathogenesis, although its detailed mechanism needs further elucidation.

As one of the first chemokines to be identified, CCL2 recruits monocyte-macrophage, basophilic, and T cells to inflammatory sites (Daly and Rollins, 2003). Our results show elevated CCL2 mRNA levels in both decidual and chorionic tissues, suggesting a role of CCL2 in the occurrence of RSA. There have been no previous studies reporting a correlation between CCL2 and RSA. CCL2 has been shown to be related to premature birth (Zhang et al., 2012), thus suggesting its involvement in pregnancy disorders. In the first trimester of pregnancy, large amounts of CCL2 are released by macrophages under the induction of fetus implantation into trophocytes, thereby recruiting and activating mononuclear-macrophages in addition to the release of cytokines for the induction of fetal nutrition and focal immune suppression (Fest et al., 2007). The elevation of CCL2 expression in decidual tissues may cause defects in macrophage recruitment and result in increased secretion of inflammatory cytokines including TNF- $\alpha$, INF- $\gamma$ and IL-1, some of which may further potentiate CCL2 expression. This would form a positive feedback loop and result in miscarriage (Lockwood et al., 2006). This study suggests the close relationship between CCL2 and RSA but the precise mechanism is unknown.

In RSA patients, elevated serum levels of TLR4 and CCL2 were found, both of which were positively correlated with their respective mRNA levels in decidual/chorionic tissues. This suggests the potential use of serum TLR4 and CCL2 levels as an index for RSA diagnosis during pregnancy. As the quantification of these two factors in either decidual or chorionic tissues involves invasive examinations, which may cause fetal injury or miscarriage, blood serum quantification has inherent advantages for evaluating the developmental status of the fetus. However, due to the limited sample size of this study, further large-scale clinical trials are required on the consistency and reproducibility of analysis of serum TLR4 and CCL2 levels in RSA patients in order to substantiate the correlation. 


\section{Conflicts of interest}

The authors declare no conflict of interest.

\section{REFERENCES}

Amirchaghmaghi E, Taghavi SA, Shapouri F, Saeidi S, et al. (2013). The role of toll like receptors in pregnancy. Int. J. Fertil. Steril. 7: 147-154.

Beijar EC, Mallard C and Powell TL (2006). Expression and subcellular localization of TLR-4 in term and first trimester human placenta. Placenta 27: 322-326.http://dx.doi.org/10.1016/j.placenta.2004.12.012

Chaouat G, Assal Meliani A, Martal J, Raghupathy R, et al. (1995). IL-10 prevents naturally occurring fetal loss in the CBA x $\mathrm{DBA} / 2$ mating combination, and local defect in IL-10 production in this abortion-prone combination is corrected by in vivo injection of IFN-tau. J. Immunol. 154: 4261-4268.

Daly C and Rollins BJ (2003). Monocyte chemoattractant protein-1 (CCL2) in inflammatory disease and adaptive immunity: therapeutic opportunities and controversies. Microcirculation 10: 247-257. http://dx.doi.org/10.1080/mic.10.3-4.247.257

Drake PM, Red-Horse K and Fisher SJ (2002). Chemokine expression and function at the human maternal-fetal interface. Rev. Endocr. Metab. Disord. 3: 159-165.http://dx.doi.org/10.1023/A:1015463130306

Dunzendorfer S, Lee HK, Soldau K and Tobias PS (2004). Toll-like receptor 4 functions intracellularly in human coronary artery endothelial cells: roles of LBP and SCD14 in mediating LPS responses. FASEB J. 18: 1117-1119.

Fest S, Aldo PB, Abrahams VM, Visintin I, et al. (2007). Trophoblast-macrophage interactions: a regulatory network for the protection of pregnancy. Am. J. Reprod. Immunol. 57: 55-66.http://dx.doi.org/10.1111/j.1600-0897.2006.00446.x

Ford HB and Schust DJ (2009). Recurrent pregnancy loss: etiology, diagnosis, and therapy. Rev. Obstet. Gynecol. 2: 76-83.

Gobert M and Lafaille JJ (2012). Maternal-fetal immune tolerance, block by block. Cell 150: 7-9. http://dx.doi.org/10.1016/j.cell.2012.06.020

Hannan NJ, Jones RL, Critchley HO, Kovacs GJ, et al. (2004). Coexpression of fractalkine and its receptor in normal human endometrium and in endometrium from users of progestin-only contraception supports a role for fractalkine in leukocyte recruitment and endometrial remodeling. J. Clin. Endocrinol. Metab. 89: 6119-6129.http://dx.doi.org/10.1210/jc.2003-031379

Hill JA, 3rd and Choi BC (2000). Immunodystrophism: evidence for a novel alloimmune hypothesis for recurrent pregnancy loss involving Th1-type immunity to trophoblast. Semin. Reprod. Med. 18: 401-406.http://dx.doi.org/10.1055/s-2000-13730

Koga K and Mor G (2010). Toll-like receptors at the maternal-fetal interface in normal pregnancy and pregnancy disorders. $A m$. J. Reprod. Immunol. 63: 587-600.http://dx.doi.org/10.1111/j.1600-0897.2010.00848.x

Koga K, Izumi G, Mor G, Fujii T, et al. (2014). Toll-like receptors at the maternal-fetal interface in normal pregnancy and pregnancy complications. Am. J. Reprod. Immunol. 72: 192-205.http://dx.doi.org/10.1111/aji.12258

Kopp EB and Medzhitov R (1999). The Toll-receptor family and control of innate immunity. Curr. Opin. Immunol. 11: 13-18. http://dx.doi.org/10.1016/S0952-7915(99)80003-X

Lin QD and Qiu LH (2010). Pathogenesis, diagnosis, and treatment of recurrent spontaneous abortion with immune type. Front. Med. China 4: 275-279.http://dx.doi.org/10.1007/s11684-010-0101-y

Lockwood CJ, Matta P, Krikun G, Koopman LA, et al. (2006). Regulation of monocyte chemoattractant protein-1 expression by tumor necrosis factor-alpha and interleukin-1beta in first trimester human decidual cells: implications for preeclampsia. Am. J. Pathol. 168: 445-452.http://dx.doi.org/10.2353/ajpath.2006.050082

Pevsner-Fischer M, Morad V, Cohen-Sfady M, Rousso-Noori L, et al. (2007). Toll-like receptors and their ligands control mesenchymal stem cell functions. Blood 109: 1422-1432.http://dx.doi.org/10.1182/blood-2006-06-028704

Saini V, Arora S, Yadav A and Bhattacharjee J (2011). Cytokines in recurrent pregnancy loss. Clin. Chim. Acta 412: 702-708. http://dx.doi.org/10.1016/j.cca.2011.01.002

Takeshita T (2004). Diagnosis and treatment of recurrent miscarriage associated with immunologic disorders: Is paternal lymphocyte immunization a relic of the past? J. Nippon Med. Sch. 71: 308-313.http://dx.doi.org/10.1272/jnms.71.308

Zhang Y, Gu Y, Li JX and Li Y (2012). Correlations between chemokines CXCL12, CCL2, RANTES and early abortion. Zhonghua Yi Xue Za Zhi 92: 9-11. 